\title{
Theory of Feedback in Clusters and Molecular Cloud Turbulence
}

\author{
Enrique Vázquez-Semadeni \\ Centro de Radioastronomía y Astrofísica, UNAM, Campus Morelia \\ P.O. Box 3-72 (Xangari), Morelia, Michoacán, México \\ email: e.vazquez@.crya.unam.mx
}

\begin{abstract}
I review recent numerical and analytical work on the feedback from both low- and high-mass cluster stars into their gaseous environment. The main conclusions are that i) outflow driving appears capable of maintaing the turbulence in parsec-sized clumps and retarding their collapse from the free-fall rate, although there exist regions within molecular clouds, and even some examples of whole clouds, which are not actively forming stars, yet are just as turbulent, so that a more universal turbulence-driving mechanism is needed; ii) outflow-driven turbulence exhibits specific spectral features that can be tested observationally; iii) feedback plays an important role in reducing the SFR; iv) nevertheless, numerical simulations suggest feedback cannot completely prevent a net contracting motion of clouds and clumps. Therefore, an appealing source for driving the turbulence everywhere in GMCs is the accretion from the environment, at all scales. In this case, feedback's most important role may be to prevent a fraction of the gas nearest to newly formed stars from actually reaching them, thus reducing the SFE.
\end{abstract}

Keywords. ISM: clouds, methods: numerical, stars: winds, outflows; turbulence

\section{Introduction}

Stars form in dense cores within molecular clouds (MCs), and simultaneously they feed back on their environment through a variety of mechanisms, including ionizing radiation, winds, outflows, and supernova explosions. This feedback crucially affects the stars' environment, in particular its physical conditions, evolution, as well as the subsequent pattern of star formation (SF). In particular, it is believed that stellar feedback may be responsible for the observed non-thermal motions in clouds and clumps, which are generally interpreted as small-scale supersonic turbulence (Zuckerman \& Evans 1974). The latter, in turn, is thought to provide support against the clouds' self-gravity, and to be able to maintain a quasi-virial equilibrium state in the clouds, thus preventing global collapse and maintaining a low global SFR. This notion is at the basis of several theoretical models of SF (e.g., McKee 1989; Matzner 2002; Krumholz \& McKee 2005; Hennebelle \& Chabrier 2008). In this contribution I review recent results on the role of feedback in driving the turbulence in MCs and their substructure, specifically on its coupling with the ambient gas, on the ability of the turbulence to support the clouds, and on its role in regulating the SFR and the SF efficiency (SFE). For a discussion on the role of feedback in the determination of the stellar masses, see the review by Bate in this volume.

\section{Background}

More than half a century ago, Oort (1954) suggested that an interstellar cycle should exist, in which the HII regions produced by the ionizing radiation of $\mathrm{O}$ stars would form a dense shell which, upon fragmenting, would produce cold cloudlets with a significant velocity dispersion. These would then grow by coalescence until they became 
gravitationally unstable, at which point they would form new stars, and the cycle would repeat. This Oort model was later formulated mathematically by Field \& Saslaw (1965), who concluded that the SFR should be proportional to the square of the gas density, in agreement with the SF law known at the time (Schmidt 1959), and that the cloud mass spectrum should scale as $M^{-3 / 2}$, is in agreement with the observed mass spectrum of CO clouds (see, e.g., Blitz 1993 and references therein) and of low-mass clumps (Motte et al. 1998). These results exemplify the fundamental implications of feedback on their environment.

Another seminal study was that of Norman \& Silk (1980, hereafter NS80), who considered the low-mass analogy of the Oort-Field-Saslaw model, with the driving energy provided in this case by the outflows produced by low-mass T-Tauri stars. In their model, the collision of expanding, wind-driven shells would form dense clumps that would then evolve under the competition of growth by coalescence, and destruction by leak, drag, or very energetic collisions.

Based on the Oort-NS80 scenario, McKee (1989) advanced a model in which MCs form from atomic cloudlets that grow by coalescence, and become molecular (in the sense that most of the carbon is in $\mathrm{CO}$ ) when the extinction $A_{V} \sim 1$. He argued that at this point the clouds must be magnetically supercritical, and therefore begin to contract as a whole, although their substructures (clumps and cores) are still subcritical, and are thus magnetically supported. Nevertheless, the contraction speeds up ambipolar diffusion (AD) in the cores, rendering them supercritical and allowing them to collapse and form stars. The latter begin driving turbulence in the cloud, eventually halting the collapse, and allowing the cloud to reach a stable equilibrium. This occurs when the mean extinction has increased to $A_{V} \sim 4-8$. In this model, the gas depletion time for typical giant MCs (GMCs) was estimated to be $\sim 2-4 \times 10^{8} \mathrm{yr}$, and the typical magnetic field strength $B \sim 20-40 \mu \mathrm{G}$.

Today, however, we know that clouds do not grow only by coalescence, but that instead a significant, and perhaps dominant, mechanism determining their mass is direct accretion from their warm, diffuse surrounding medium, allowing for much shorter growth timescales (Blitz \& Shu 1980; Hennebelle \& Pérault 1999; Ballesteros-Paredes et al. 1999a,b; Koyama \& Inutsuka 2002; Audit \& Hennebelle 2005; Hennebelle \& Audit 2007; Vázquez-Semadeni et al. 2006; Banerjee et al. 2009), and that this mechanism can drive strong turbulence in the clouds. Thus, more recent research has focused on the details of the physics that regulates the energy transfer from the stellar sources, especially bipolar outflows from low-mass stars, to their environment, as well as to whether indeed stellar feedback is capable of feeding the clouds' turbulence and maintaining them in rough hydrostatic equilibrium. I now briefly summarize some of the main results in these respects.

\section{Feedback from outflows}

\subsection{Efficiency of coupling with the environment}

Estimates of the amount of energy injected to the environment by an outflow $\left(\sim 10^{47}\right.$ erg per $M_{\odot}$; Shu et al. 1988) suggest that outflows may deposit enough kinetic energy in their parent clump as to maintain its turbulence (see Reipurth \& Bally 2001 and references therein). However, an important, recurrent question is whether the bipolar outflows couple efficiently to their environment.

In this regard, Quillen et al. (2005) investigated the correlation of the molecular gas kinematics in NGC1333 with the distribution of young stellar objects (YSOs) within this 
cloud, finding that the velocity dispersion does not vary significantly across the cloud and is uncorrelated with the number of nearby young stellar outflows. However, they did find about 20 cavities in the velocity channel maps that they interpreted as remnants of past outflow activity. Those authors concluded that, while outflows may not directly drive the turbulence in the clumps, the cavities ("fossil outflows") may provide an efficient coupling mechanism to the environment. Later numerical work by Cunningham et al. (2006b) supported this conclusion.

The interaction of single, or a few, outflows with their environment has been extensively studied. De Colle \& Raga (2005) performed magnetohydrodynamic (MHD) simulations of high-density clumps propagating through a high-density medium, in order to represent the interaction of a jet with the medium. They found that $\sim 10-30 \%$ of the jet's kinetic energy can be transferred to the medium, depending on the magnetic field's strength and orientation relative to the jet. Cunningham et al. (2006a) investigated the effect of collisions among outflows, finding that the collisions reduce the efficiency of transfer to the cloud, so that the most efficient drivers are isolated outflows. However, Banerjee et al. (2007) noted, using isothermal simulations, that outflows should be inefficient drivers of supersonic turbulent motions in their parent clouds because the compressions they produce can only re-expand sonically, although then Cunningham et al. (2009) argued that this problem can be circumvented if the cloud is previously turbulent (i.e, the turbulence is maintained rather than generated), and adequate care is taken of modeling the cooling.

It can be concluded from this section that, through the mediation of long-lived cavities, magnetic fields, and a pre-existent turbulent velocity field, outflows appear capable of at least maintaining the turbulent motions in a clump.

\subsection{Feedback and nature of the turbulence}

In real MCs, stars are almost always born in clusters, and so the effect of an ensemble of outflows on their parent clump is also a crucial issue. In particular, many studies have focused on the nature of the turbulent motions induced in the parent clump by an ensemble of outflows. We now turn to this issue.

Numerical simulations of evolving clumps at the parsec scale suggest that the initial ("interstellar") turbulence is quickly replaced by "outflow" turbulence, at least within the scales modeled by these simulations (Li \& Nakamura 2006; Nakamura \& Li 2007). This transition consists in a secular variation of the topology of the density and velocity fields in the clump. The density field develops a central concentration, with a power-law profile of the form $\rho \sim r^{-3 / 2}$, and the velocity field develops a circulation pattern, with gravity driving infall motions that balance the outward motions driven by the outflows. Moreover, the turbulent energy spectrum develops a knee at a characteristic "outflow scale" (Matzner 2007), and a slope steeper than that of isotropic random driving at scales smaller than this (Nakamura \& Li 2007; Carroll et al. 2009, 2010).

A particularly interesting feature is that the presence of the outflows does not seem to impede the development of coherent streams of infalling gas towards the center of the gravitational potential well (Nakamura \& Li 2007). This implies that the most massive stars forming in the clump are fed from a mass supply that extends out to the scale of the whole clump, instead of being restricted to the scale of the very dense core that contains the forming star (Wang et al. 2010). This result is in stark contrast with the currently popular notion that the masses of forming stars are determined by the masses of the cores in which they reside (e.g., Padoan \& Nordlund 2002, Krumholz et al. 2005, Alves et al. 2007), and more in agreement with the scenario of competitive accretion for star formation (e.g., Bonnell et al. 1997; Bonnell \& Bate 2006; Bonnell et al. 2007; Smith 
et al. 2009), in which the material reaching a star is collected from distant locations (up to several tenths of a parsec away) within the clump. In fact, the development of a density profile with slope $-3 / 2$ may also be indicative of a generalized state of collapse in the clumps, as it is the signature of ongoing dynamic collapse (Shu 1977).

In summary, the simulations discussed above suggest that outflow feedback is capable of maintaining the turbulence within parsec-scale clumps that are already forming stars. However, there exist a few observational features of clouds that cannot be explained by this mechanism. First, it is well known that the majority of a cloud's volume is not in the process of forming stars; the star-forming regions within clouds are generally limited to only a few localized, high-column density spots (e.g, Kirk et al. 2006) within the clouds. Moreover, there exist clouds with very little or no significant star-forming activity, such as Maddalena's cloud (Maddalena \& Thaddeus 1985) or the Pipe Nebula (Onishi et al. 1999; see also Lombardi et al. 2006) that nevertheless have turbulent properties essentially indistinguishable from those of clouds that are actively forming stars. Outflow feedback clearly cannot explain the origin or maintenance of the turbulence in the non-star-forming regions.

Second, principal component analysis (PCA) of spectroscopical line emission data (Heyer \& Brunt 2007; Brunt et al. 2009) shows that the turbulent velocity dispersion in the clouds and their substructure appears to be dominated by large-scale, dipolar, velocity gradients spanning the entire structure. Such universal large-scale nature of the turbulent motions in clouds does not seem to be attainable by outflows, due to their small-scale, localized nature. Note, however, that Carroll et al. (2010) have recently questioned these results, a claim that requires further investigation. In any case, the problem of driving the turbulence in non-star-forming regions remains, and in general it can be argued that a different source of turbulence is required there.

\section{Cloud evolution and control of the SFE}

\subsection{Cloud destruction}

The expansion of HII regions from massive stars, especially from those that are sufficiently close to the cloud's periphery to produce a "blister HII region", is probably the dominant feedback mechanism at the scale of GMCs (for a discussion of the contributing feedback mechanisms, see Matzner 2002), and is generally believed to be capable of effectively dispersing the cloud within $10^{7}$ yr after SF starts in the cloud (Blitz \& Shu 1980). Whitworth (1979) analytically estimated the eroding effect of O stars producing blister HII regions in MCs, and concluded that the cloud would be completely dispersed after only $4 \%$ of its mass had been converted to stars, assuming a standard Salpeter (1955) initial mass function (IMF).

Extending on this result, and considering that a fraction of the massive stars are completely interior to the cloud and do not produce a blister HII region, Franco et al. (1994) estimated the maximum number of OB stars that can be hosted by a GMC without it being destroyed, concluding that the resulting SFE should range between $2 \%$ and $16 \%$, with an average of $5 \%$. On the observational side, Williams \& McKee (1997) compared the Galactic distribution of GMC masses to the distribution of OB association luminosities, in order to statistically estimate the GMC mass that contains at least one $\mathrm{O}$ star. They found that the median GMC mass to satisfy this condition is $\sim 10^{5} M_{\odot}$, and that the average SFE is $\sim 5 \%$. Also, they estimated that the typical GMC lifetime as the time for the cloud to be photo-evaporated by the $\mathrm{O}$ stars is $\sim 3 \times 10^{7} \mathrm{yr}$, although a 
more recent estimate by Matzner (2002) yields a somewhat shorter timescale of $\sim 2 \times 10^{7}$ yr.

\subsection{Support of isolated clouds and the SFE}

Together with the erosion inflicted on clouds by massive-star feedback, it is also generally believed that the latter can also maintain GMCs close to virial equilibrium for times significantly longer than their free-fall times. Taking this as a working assumption, the SFR and SFE can be derived, similarly to the procedure used in studies of SF selfregulation due to outflows (e.g., Franco \& Cox 1983; McKee 1989). For example, Matzner (2002) analytically derived a cloud destruction timescale of $\sim 2 \times 10^{7}\left(M_{\mathrm{cl}} / 10^{6} M_{\odot}\right)^{-1 / 3}$ yr, implying that more massive clouds should be destroyed in shorter times.

A semi-analytic model of the energy balance in GMCs was presented by Krumholz et al. (2006). In this model, the fully time-dependent Virial Theorem was written and solved numerically for a spherical cloud under the influence of its self-gravity and the HII-region feedback, with no a-priori assumption of equilibrium. The result was that clouds undergo a few expansion-contraction oscillations, until they are finally dispersed, with lower-mass clouds $\left(M \sim 2 \times 10^{5} M_{\odot}\right)$ are more quickly dispersed (typically within $\sim 1.5$ crossing times) than more massive ones $\left(M \sim 5 \times 10^{6} M_{\odot}\right)$, which last $\sim 3$ crossing times. Note that this result is opposite to that of Matzner (2002). The SFEs over the clouds' lifetimes were found to be $\sim 5-10 \%$.

Full numerical simulations including self-consistent SF prescriptions, aimed at studying the role of stellar feedback on reducing the SFR have been generally carried out at the clump-scale level. There is a general agreement that feedback reduces the SFR and, consequently, the SFE of a cloud, regardless of whether the energy is injected by low-mass outflows (Nakamura \& Li 2007; Wang et al. 2010), low-mass protostellar luminosity (Bate 2009; Price \& Bate 2009; Offner et al. 2009), or high-mass-star winds (Dale \& Bonnell 2008). Although the details vary depending on the specific implementation, all studies conclude that the SFR is reduced with respect to the case with no feedback. In particular, some of these studies quantify the SFE, reporting values $\lesssim 10 \%$ per free-fall time (e.g., Wang et al. 2010), in agreement with observations of similar regions (e.g., Evans et al. 2009), although Dale \& Bonnell (2008) warn that the the SFR may accelerate in time, rendering any conclusions based on the average SFE uncertain.

\subsection{Evolution of cloud-environment systems}

All the numerical studies mentioned in the previous section have been performed at the clump scale, thus omitting the interaction between the clumps and the larger-scale cloud in which they are immersed. This may be of crucial importance, since recent simulations of GMC formation and evolution in the presence of self-gravity (Vázquez-Semadeni et al. 2007, 2009; Hartmann \& Burkert 2007; Heitsch \& Hartmann 2008; Heitsch et al. 2008; Hennebelle et al. 2008; Banerjee et al. 2009; see also the review by Vázquez-Semadeni 2010) as well as observations of massive-star forming regions (Galván-Madrid et al. 2009; Csengeri et al. 2010) suggest that there is a continuous infalling flow from the large to the small scales, most probably driven by gravity. In this case, the mass reservoir of the local star-forming regions is not limited to its immediate environment, but rather includes material from regions farther away in the cloud than the local clump.

A numerical study incorporating the effect of massive-star ionizing radiation in the context of globally contracting clouds was recently performed by Vázquez-Semadeni et al. (2010). These authors considered the evolution of clouds formed by converging flows in the warm neutral atomic medium (WNM), and followed it until the time of active SF. In this type of simulations, the clouds are found to enter a global state of contraction, 
causing the localized star-forming regions within the clouds to have a continuous inflow of mass from their environment, rather than having a fixed mass. Thus, the SFE, defined as $\mathrm{SFE}=M_{\star} /\left(M_{\mathrm{cl}}+M_{\star}\right)$, where $M_{\star}$ is the total mass in stars and $M_{\mathrm{cl}}$ is the gas mass of the cloud, can maintain realistic values, of order of a few percent for GMCs, over extended periods of time, because the gas mass is replenished by the infall while the cloud continues forming stars. These authors also found that the feedback acts on size scales much smaller than the gravitational potential well of the whole GMC, and therefore the global inflow persists, even if locally the gas on route to forming stars is dispersed, reducing the SFE. This effect is stronger for more massive clouds, which have deeper and more extended potential wells. Smaller clouds were found to be more easily destroyed, in agreement with the semi-analytic model of Krumholz et al. (2006). However, the results from Vázquez-Semadeni et al. (2010) suggest that perhaps termination of the SF activity on the scale of the largest GMCs may require the termination of the inflows, rather than being accomplished by the feedback. Further exploration of parameter space and feedback modeling is needed in order to obtain firmer conclusions in this regard.

The possibility that clouds are in a generalized state of contraction and accreting from WNM has the additional advantage that it may provide the needed universal source of turbulence in GMCs and their substructure, since it is by now well established that the dense layers produced by converging flows naturally develop turbulence, as a consequence of several instabilities acting on them (Hunter et al. 1986; Vishniac 1994; Folini \& Walder 2006; Heitsch et al. 2006; Vázquez-Semadeni et al. 2006). Recently, Klessen \& Hennebelle (2009) have compared the energy input rate from the accretion to the energy dissipation rate by the turbulence, concluding that the former is sufficient to maintain the turbulence even if only $10 \%$ of the accretion energy is converted to turbulence in the dense regions. These authors suggest that this mechanism can operate at all scales from Galactic disks to protostellar disks, passing through GMCs and their substructure, extending the suggestion that a universal mass cascade, driven by gravity, exists at all scales within GMCs (Field et al. 2008).

\section{Discussion and conclusions}

The results from the works discussed in this review imply that feedback from clusters has a complex and strongly nonlinear effect on their parent clouds and clumps, which still remains elusive in some respects. Numerical simulations of outflows from low-mass stars in parsec-sized clumps agree in general that the outflows inject sufficient momentum into the clump to sustain the turbulence in it. In these simulations, the turbulence develops a peculiar form of the turbulent energy spectrum, with a knee at the characteristic outflow scale, and a steep slope $(\sim-2.5)$ below that scale. These features should in principle be observationally detectable, and so this prediction is directly testable. However, we pointed out that outflow driving cannot account for the turbulence in non-star-forming regions of clouds, or in clouds that have no significant star-forming activity at all. Thus, we concluded that a more universal source of turbulence is needed.

Analytical and semi-analytical calculations of the effect of feedback from massive stars at the GMC scale suggest that the feedback is also capable of slowing down the SFR to realistic rates, while simultaneously supporting the cloud over a few crossing times, after which the cloud is finally destroyed. However, by their very nature, these models cannot account for the spatial distribution of the stellar sources, an ingredient which is suggested to be crucial by numerical studies of the formation and evolution of a large molecular complex. Such simulations show that the entire cloud complex begins contracting gravitationally even before it becomes predominantly molecular, so that, by the time 
a GMC is fully formed and begins to form stars, it is already contracting. Moreover, the contraction occurs in a highly non-uniform fashion, due to the turbulence produced at the cloud's formation, so that SF and their feedback occur at a few localized spots in the clouds. This prevents the feedback from reaching the more distant, yet also infalling, regions of the clouds.

This suggests again that the source of the turbulence at the scale of whole GMCs should be a more universal one than the feedback, which is applied very locally. Within this scenario, a natural candidate source is the accretion energy from the environment, since it is well known that the dense layers formed by colliding streams are naturally turbulent due to several dynamical instabilities acting on them. In this picture, the GMCs are the dense "layers" within converging WNM flows, while clumps and cores are the dense "layers" within converging molecular and/or cold atomic convergent flows, all probably driven by gravity rather than by the stellar feedback. The latter is only a byproduct of the gravitational contraction, and acts mainly to prevent a fraction of the infalling gas mass from actually reaching the forming stars, reducing the SFR and the SFE from their free-fall value. More theoretical, numerical and observational work is clearly needed to confirm this picture, and sort out its details.

\section{References}

Alves, J., Lombardi, M., \& Lada, C. J. 2007, A\&广A, 462, L17

Audit, E. \& Hennebelle, P. 2005, A\&A, 433, 1

Ballesteros-Paredes, J., Vázquez-Semadeni, E., \& Scalo, J. 1999, ApJ, 515, 286

Ballesteros-Paredes, J., Hartmann, L., \& Vázquez-Semadeni, E. 1999, ApJ, 527, 285

Banerjee, R., Klessen, R. S., \& Fendt, C. 2007, ApJ, 668, 1028

Banerjee, R., Vázquez-Semadeni, E., Hennebelle, P., \& Klessen, R. S. 2009, MNRAS, 398, 1082

Bate, M. R. 2009, MNRAS, 392, 1363

Blitz, L. 1993, Protostars and Planets III, 125

Blitz, L. \& Shu, F. H. 1980, ApJ, 238, 148

Bonnell, I. A. \& Bate, M. R. 2006, MNRAS, 370, 488

Bonnell, I. A., Bate, M. R., Clarke, C. J., \& Pringle, J. E. 1997, MNRAS, 285, 201

Bonnell, I. A., Larson, R. B., \& Zinnecker, H. 2007, Protostars and Planets V, 149

Brunt, C. M., Heyer, M. H., \& Mac Low, M.-M. 2009, A $\& A$ A, 504, 883

Carroll, J. J., Frank, A., Blackman, E. G., Cunningham, A. J., \& Quillen, A. C. 2009, ApJ, 695, 1376

Carroll, J. J., Frank, A., \& Blackman, E. G. 2010, ApJ, 722, 145

Csengeri, T., Bontemps, S., Schneider, N., Motte, F., \& Dib, S. 2010, arXiv:1009.0598

Cunningham, A. J., Frank, A., \& Blackman, E. G. 2006a, ApJ, 646, 1059

Cunningham, A. J., Frank, A., Quillen, A. C., \& Blackman, E. G. 2006b, ApJ, 653, 416

Cunningham, A. J., Frank, A., et al. 2009, ApJ, 692, 816

Dale, J. E. \& Bonnell, I. A. 2008, MNRAS, 391, 2

De Colle, F. \& Raga, A. C. 2005, MNRAS, 359, 164

Evans, N. J., et al. 2009, ApJS, 181, 321

Field, G. B., Blackman, E. G., \& Keto, E. R. 2008, MNRAS, 385, 181

Field, G. B. \& Saslaw, W. C. 1965, ApJ, 142, 568

Folini, D. \& Walder, R. 2006, A\&SA, 459, 1

Franco, J. \& Cox, D. P. 1983, ApJ, 273, 243

Franco, J., Shore, S. N., \& Tenorio-Tagle, G. 1994, ApJ, 436, 795

Galván-Madrid, R., Keto, E., Zhang, Q., Kurtz, S., Rodríguez, L. F., \& Ho, P. T. P. 2009, ApJ, 706, 1036

Hartmann, L. \& Burkert, A. 2007, ApJ, 654, 988

Heitsch, F., Burkert, A., Hartmann, L. W., Slyz, A. D., \& Devriendt, J. E. G. 2005, ApJ, 633, L113 
Heitsch, F. \& Hartmann, L. 2008, ApJ, 689, 290

Heitsch, F., Hartmann, L. W., Slyz, A. D., Devriendt, J. E. G., \& Burkert, A. 2008, ApJ, 674, 316

Heitsch, F., Slyz, A. D., Devriendt, J. E. G., Hartmann, L. W., \& Burkert, A. 2006, ApJ, 648, 1052

Hennebelle, P. \& Audit, E. 2007, A\&A, 465, 431

Hennebelle, P., Banerjee, R., Vázquez-Semadeni, E., Klessen, R. S., \& Audit, E. 2008, A\&A, 486, L43

Hennebelle, P. \& Chabrier, G. 2008, ApJ, 684, 395

Hennebelle, P. \& Pérault, M. 1999, A\&A, 351, 309

Heyer, M. H. \& Brunt, C. 2007, IAU Symposium 237, 9

Hunter, J. H., Jr., Sandford, M. T., II, Whitaker, R. W., Klein, R. I. 1986, ApJ, 305, 309

Kirk, H., Johnstone, D., \& Di Francesco, J. 2006, ApJ, 646, 1009

Klessen, R. S. \& Hennebelle, P. 2009, A\&A in press (arXiv:0912.0288)

Koyama, H. \& Inutsuka, S.-i. 2002, ApJ, 564, L97

Krumholz, M. R., Matzner, C. D., \& McKee, C. F. 2006, ApJ, 653, 361

Krumholz, M. R. \& McKee, C. F. 2005, ApJ, 630, 250

Krumholz, M. R., McKee, C. F., \& Klein, R. I. 2005, Nature, 438, 332

Li, Z.-Y. \& Nakamura, F. 2006, ApJ, 640, L187

Lombardi, M., Alves, J., \& Lada, C. J. 2006, Aछ A, 454, 781

Maddalena, R. J. \& Thaddeus, P. 1985, ApJ, 294, 231

Matzner, C. D. 2002, ApJ, 566, 302

Matzner, C. D. 2007, ApJ, 659, 1394

McKee, C. F. 1989, ApJ, 345, 782

Motte, F., Andre, P., \& Neri, R. 1998, A\&A, 336, 150

Nakamura, F. \& Li, Z.-Y. 2007, ApJ, 662, 395

Norman, C. \& Silk, J. 1980, ApJ, 238, 158

Offner, S. S. R., Klein, R. I., McKee, C. F., \& Krumholz, M. R. 2009, ApJ, 703, 131

Onishi, T., et al. 1999, PASJ, 51, 871

Oort, J. H. 1954, Bull. Astron. Inst. Netherlands, 12, 177

Padoan, P. \& Nordlund, A. 2002, ApJ, 576, 870

Price, D. J. \& Bate, M. R. 2009, MNRAS, 398, 33

Quillen, A. C., Thorndike, S. L., Cunningham, A., Frank, A., Gutermuth, R. A., Blackman, E. G., Pipher, J. L., \& Ridge, N. 2005, ApJ, 632, 941

Reipurth, B. \& Bally, J. 2001, ARAA, 39, 403

Salpeter, E. E. 1955, ApJ, 121, 161

Schmidt, M. 1959, ApJ, 129, 243

Shu, F. H. 1977, ApJ, 214, 488

Shu, F. H., Lizano, S., Ruden, S. P., \& Najita, J. 1988, ApJL, 328, L19

Smith, R. J., Clark, P. C., \& Bonnell, I. A. 2009, MNRAS, 396, 830

Vazquez-Semadeni, E. 2010, in The Dynamic ISM: A celebration of the Canadian Galactic Plane Survey, ASP Conference Series (arXiv:1009.3962)

Vázquez-Semadeni, E., Colín, P., Gómez, G. C., Ballesteros-Paredes, J., \& Watson, A. W. 2010, ApJ, 715, 1302

Vázquez-Semadeni, E., Gómez, G. C., Jappsen, A. K., Ballesteros-Paredes, J., González, R. F., \& Klessen, R. S. 2007, ApJ, 657, 870

Vázquez-Semadeni, E., Gómez, G. C., Jappsen, A.-K., Ballesteros-Paredes, J., \& Klessen, R. S. 2009, ApJ, 707, 1023

Vázquez-Semadeni, E., Ryu, D., Passot, T., González, R. F., \& Gazol, A. 2006, ApJ, 643, 245

Vishniac, E. T. 1994, ApJ, 428, 186

Wang, P., Li, Z.-Y., Abel, T., \& Nakamura, F. 2010, ApJ, 709, 27

Whitworth, A. 1979, MNRAS, 186, 59

Williams, J. P. \& McKee, C. F. 1997, ApJ, 476, 166

Zuckerman, B. \& Evans, N. J., II 1974, ApJL, 192, L149 